\title{
On a reaction-diffusion system involving the critical exponent.
}

\author{
Nicolae TARFULEA
}

\begin{abstract}
In this paper we study the existence and multiplycity of the nontrivial solutions for the following elliptic system with Dirichlet boundary conditions and critical nonlinearity

$$
\left\{\begin{array}{ll}
-\Delta u=\lambda u+W(x) u|u|^{2^{+}-2}-k v & \text { in } \Omega \\
-\Delta v=\delta u-\gamma v & \text { in } \Omega \\
u=v=0 & \text { on } \partial \Omega
\end{array},\right.
$$

where $\Omega \subset \mathbf{R}^{N}(N \geq 3)$ is a bounded regular domain, $W(.) \epsilon$ $L^{\infty}(\Omega)$ with the property that there exists $\eta>0$ such that $W(\cdot) \geq$ $\eta$ a.e. in $\Omega$ and $\lambda, \delta, \gamma$ are real parameters. We show that the number of nontrivial solutions, in a left neighbourhood of each $\widehat{\lambda_{j}}, j=1,2, \ldots$, is at least twice the multiplicity of $\widehat{\lambda_{j}}$, where the set $\left\{\widehat{\lambda_{j}}\right\}_{j \in \mathbb{N}^{*}}$ represents the spectrum of a certain integrodifferential operator.
\end{abstract}

\section{Introduction}

Rothe in $[R]$ considered the system of reaction diffusion equations

$$
\left\{\begin{array}{l}
\partial u \partial t=\mu \Delta u+f(u)-v \\
\varepsilon \partial v \partial t=\Delta v+u-v
\end{array}\right.
$$

1991 Mathematics Subject Classification: 35J55, 35J50.

Partially supported by a CNCSU-Grant $n^{0}$ 195/1997.

Servicio Publicaciones Univ. Complutense. Madrid, 1998. 
for $(t, x) \in(0, \infty) \times \Omega$. Here $u, v$ are real functions of $(t, x) \in[0, \infty) \times \bar{\Omega}$, where $\Omega \subset \mathbf{R}^{N}(N \geq 1)$ is open, bounded and connected. As explained in [RM], $u$ and $v$, which are called the activator and inhibitor respectively, can be interpreted as relative concentrations of substances known as morphogens. The system (1) is supplemented by Dirichlet boundary conditions

$$
u=v=0, \text { for }(t, x) \in(0, \infty) \times \partial \Omega
$$

and the initial conditions

$$
u(0, x)=u_{0}(x), v(0, x)=v_{0}(x), \text { for all } x .
$$

As shown in [RM], the existence of equilibrium solutions in (1) is determined by the problem with $\varepsilon=0$ and the equilibrium states are solutions of the elliptic system

$$
\begin{cases}\mu \Delta u+f(u)-v=0 & \text { in } \Omega \\ \triangle v+u-v=0 & \text { in } \Omega\end{cases}
$$

subject to Dirichlet boundary conditions

$$
u=v=0 \text { on } \partial \Omega .
$$

It will be convenient to split the function $f$, which models autocatalytic and saturation effects, into the linear and higher order terms

$$
f(u)=\lambda u+g(u) .
$$

Notation. In the rest of the paper we make use of the following notation $L^{p}(\Omega), 1 \leq p \leq \infty$, denote Lebesgue spaces; the norm in $L^{p}$ is denoted by $\|\cdot\|_{p}$

$W^{k, p}(\Omega)$ denote Sobolev spaces;

$H_{0}^{1}(\Omega)$ denotes $W_{0}^{1,2}(\Omega)$, endowed with the norm $\|u\|^{2}=\Omega|\nabla u|^{2} \mathrm{~d} x$;

$H^{-1}(\Omega)$ denotes the topological dual of $H_{0}^{1}(\Omega)$; the norm in this space is denoted by $\|\cdot\|_{H^{-1}}$.

We consider below the problem of finding nontrivial solutions of the slightly more general elliptic system with Dirichlet boundary conditions and critical nonlinearity

$$
\left\{\begin{array}{ll}
-\Delta u=\lambda u+W(x) u|u|^{2^{*}-2}-k v & \text { in } \Omega \\
-\Delta v=\delta u-\gamma v & \text { in } \Omega \\
u=v=0 & \text { on } \partial \Omega
\end{array},\right.
$$


where $\Omega \subset \mathbf{R}^{N}(N \geq 3)$ is a bounded regular domain, $\delta, \gamma$ and $k$ are constants such that $\bar{k} \delta>0$ and $\gamma>-\lambda_{1}(\Omega)$, where $\lambda_{1}(\Omega)$ is the first eigenvalue of the Dirichlet Laplacian on $\Omega$, and $W(\cdot) \in L^{\infty}(\Omega)$ with the property that there exists $\eta>0$ such that $W(\cdot) \geq \eta$ a.e. in $\Omega$. Here $2^{*}=2 N N-2$.

In the subcritical case the system (1) has been studied by various authors (see [Ro], [Si], [FM], [NT] and others). The review, even partial, of their results is out of the scope of this paper.

Assuming $u$ to be known, the Dirichlet boundary value problem

$$
\begin{cases}-\Delta v+\gamma v=\delta u & \text { in } \Omega \\ v=0 & \text { on } \partial \Omega\end{cases}
$$

is uniquely solved by $v=1 k B u$ where the operator $B=k \delta(-\triangle+\gamma)^{-1}$ is bounded from $L^{p}(\Omega)$ to $W^{2, p}(\Omega)$ for all $1 \leq p<\infty$. Also, by the Schauder theory, $B$ maps the Hölder space $C^{\alpha}(\bar{\Omega})$ into $C^{1+\alpha}(\bar{\Omega})$.

Moreover, it is easily checked that $B$ is positive and self-adjoint in the sense that

$$
\int_{\Omega} u B u \mathrm{~d} x=\frac{1}{k \delta} \int_{\Omega}|\nabla w|^{2}+\gamma w^{2} \mathrm{~d} x
$$

for $u \in L^{2}(\Omega)$ and $w=B u$; and if $w=B u, z=B v$ then

$$
\int_{\Omega} u B v d x=\frac{1}{k \delta} \int_{\Omega} \nabla w \nabla z+\gamma w z \mathrm{~d} x=\int_{\Omega} v B u \mathrm{~d} x .
$$

Let us define the operator

$$
T \equiv-\triangle+B: L^{2}(\Omega) \rightarrow L^{2}(\Omega) \text {, with } D(T)=W^{2,2}(\Omega) \cap H_{0}^{1}(\Omega) \text {. }
$$

It is easy to observe that $T$ is symmetric on its domain $D(T)$ i.e.

$$
\left\langle T u_{1}, u_{2}\right\rangle=\left\langle u_{1}, T u_{2}\right\rangle \text { for all } u_{1}, u_{2} \in D(T),
$$

where $\langle\cdot, \cdot\rangle$ denotes the $L^{2}$-inner product.

If $0<\lambda_{1}<\lambda_{2} \leq \lambda_{3} \leq \ldots$ and $\left(\varphi_{k}\right)_{k}$ denote respectively the eigenvalues and the eigenfunctions of $-\Delta$ in $\Omega$ under zero Dirichlet boundary conditions, then one can verify easily that the $\varphi_{k}$ 's are also eigenfunctions of $T$ corresponding to the modified eigenvalues

$$
\widehat{\lambda_{k}}=\lambda_{k}+\frac{k \delta}{\gamma+\lambda_{k}}, k=1,2, \ldots
$$


A more detailed analysis shows that the spectrum $\sigma(T)$ of $T$ consists precisely of these eigenvalues (see [FM, Corollary 1.2.]).

From the above, we obtain that $(\mathrm{P})$ is equivalent to the integrodifferential equation

$$
\left\{\begin{array}{lc}
-\triangle u+B u=\lambda u+W(x) u|u|^{2^{*}-2} & \text { in } \Omega \\
u=0 & \text { on } \partial \Omega
\end{array} .\right.
$$

We associate to the problem ( $\left.\mathrm{P}^{\prime}\right)$ the functional

$$
I_{\lambda}(u)=\frac{1}{2} \int_{\Omega}|\nabla u|^{2}+u B u-\lambda u^{2} \mathrm{~d} x-\frac{1}{2^{*}} \int_{\Omega} W(x)|u|^{2^{*}} \mathrm{~d} x, \forall u \in H_{0}^{1}(\Omega) .
$$

In a standard way we can prove that $I_{\lambda} \in C^{1}\left(H_{0}^{1}(\Omega), \mathbf{R}\right)$ and the critical points of $I_{\lambda}$ are solutions of $\left(\mathrm{P}^{\prime}\right)$.

Note that $p=2^{*}$ is the limiting Sobolev exponent for the embedding $H_{0}^{1}(\Omega) \hookrightarrow L^{p}(\Omega)$. Since this embedding is not compact, the functional $I_{\lambda}$ does not satisfy the Palais-Smale condition in the energy range $(-\infty,+\infty)$. Hence there are serious difficulties when trying to find critical points by standard variational methods.

Using the ideas of Pohozaev (see [P]), Figueiredo and Mitidieri obtained a similar identity for the system (P) (see [FM, Lemma 4.1 and Remark 2.7]). From this identity, if $\Omega$ is starshaped, we can obtain that (P) admits only the trivial solution $u \equiv v \equiv 0$ for $\lambda \leq 0$.

Denote

$$
S_{B}=\inf _{u \in H_{0}^{1}(\Omega) \backslash\{0\}} \frac{\|u\|_{B}^{2}}{\|u\|_{2^{*}}^{2}}
$$

where $\|u\|_{B}^{2}=\Omega|\nabla u|^{2}+u B u \mathrm{~d} x, \forall u \in H_{0}^{1}(\Omega)$. From the positivity of $B$ we have that

$$
S_{B} \geq S=\inf _{u \in H_{0}^{1}(\Omega) \backslash\{0\}} \frac{\|u\|_{1}^{2}}{\|u\|_{2^{*}}^{2}}
$$

where $S$ corresponds to the best constant for the Sobolev continuous embedding $H_{0}^{1}(\Omega) \hookrightarrow L^{2^{*}}(\Omega)$. Then $S_{B}>0$ because it is well known that $S>0$.

Under the above conditions and notations, the result proved in this paper is the following: 
Theorem 1.1. For $\lambda>0$ denote $\widehat{\lambda_{+}}=\min \left\{\widehat{\lambda_{j}}: \lambda<\widehat{\lambda_{j}}\right\}$ and suppose that the multiplicity of $\widehat{\lambda_{+}}$is $m$. Then, if

$$
\widehat{\lambda_{+}}-\lambda<\left(\frac{\eta}{\|W\|_{\infty}}\right)^{\frac{2}{2^{+}}} S_{B}[\operatorname{meas}(\Omega)]^{-2 / N}
$$

the problem $(P)$ admits at least $m$ pairs of nontrivial solutions

$$
\left\{\left(u_{k}(\lambda), v_{k}(\lambda)\right) ;\left(-u_{k}(\lambda),-v_{k}(\lambda)\right)\right\}, k=1,2, \ldots, m .
$$

Moreover

$$
\left\|u_{k}(\lambda)\right\| \rightarrow 0 \text { and }\left\|v_{k}(\lambda)\right\| \rightarrow 0, \text { as } \lambda \nearrow \widehat{\lambda_{+}}
$$

for every

$$
k \in\{1,2, \ldots, m\}
$$

The proof of the above theorem uses standard ideas and the techniques are essentially the same as those used in [CFS] and [CFP]. The main tool used is the following slightly modified result of Bartolo, Benci and Fortunato (see [BBF, Theorem 2.4]) contained in [CFS, Theorem $2.5]$ :

Theorem 2.2. Let $H$ be a real Hilbert space with norm $\|\cdot\|_{H}$ and suppose $I \in C^{1}(H, \mathbf{R})$ is a functional on $H$ satisfying the following conditions:

I1) $I$ is even, $I(0)=0$;

I2) There exists a constant $\beta>0$ such that the Palais-Smale condition (PS) holds in $(0, \beta)$;

I3) There exist two closed subspaces $V, W \subset H$ and positive constants $\rho, \xi, \beta^{\prime}$ with $\xi<\beta^{\prime}<\beta$ such that

i) $I(u) \leq \beta^{\prime}$ for any $u \in W$;

ii) $I(u) \geq \xi$ for any $u \in V,\|u\|_{H}=\rho$;

iii) codim $V<\infty$ and $\operatorname{dim} W \geq \operatorname{codim} V$.

Then there exists at least $\operatorname{dim} W-\operatorname{codim} V$ pairs of critical points of $I$ with critical values belonging to the interval $\left[\xi, \beta^{\prime}\right]$. 


\section{Proof of Theorem 1}

\section{Step1.}

First we show that although the Palais-Smale condition does not hold globally for $I_{\lambda}$ it is satisfied locally in $\left(-\infty, 1 N S_{B}^{N / 2}\|W\|_{\infty}^{\frac{N-2}{2}}\right)$ in the following sense:

If $c<1 N S_{B}^{N / 2}\|W\|_{\infty}^{\frac{N-2}{2}}$ and $\left(u_{m}\right)_{m \geq 1}$ is a sequence in $H_{0}^{1}(\Omega)$ such that

$$
\left\{\begin{array}{l}
I_{\lambda}\left(u_{m}\right) \rightarrow c \\
\mathrm{~d} I_{\lambda}\left(u_{m}\right) \rightarrow 0 \text { strongly in } H^{-1}(\Omega)
\end{array} \text {, as } m \rightarrow \infty,\right.
$$

then $\left(u_{m}\right)_{m>1}$ contains a subsequence converging strongly in $H_{0}^{1}(\Omega)$.

Let $c \in\left(-\infty, 1 N S_{B}^{N / 2}\|W\|_{\infty}^{\frac{N-2}{2}}\right)$ and let $\left(u_{m}\right)_{m \geq 1} \subset H_{0}^{1}(\Omega)$ be a sequence such that

$$
\begin{aligned}
I_{\lambda}\left(u_{m}\right) & \rightarrow c, \text { as } m \rightarrow \infty, \text { and } \\
\mathrm{d} I_{\lambda}\left(u_{m}\right) & \rightarrow 0, \text { as } m \rightarrow \infty, \text { in } H^{-1}(\Omega)
\end{aligned}
$$

It is easy to observe that there exists $M>0$ a positive constant such that, for every $m \in \mathbf{N}^{*},\left|I_{\lambda}\left(u_{m}\right)\right| \leq M$.

If we choose $\theta \in\left(12^{*}, 12\right)$ and $m \in \mathbf{N}^{*}$ sufficiently large, we obtain

$$
\begin{gathered}
M+\theta|| u_{m} \| \geq I_{\lambda}\left(u_{m}\right)-\theta \mathrm{d} I_{\lambda}\left(u_{m}\right) u_{m} \geq \frac{1}{2} \int_{\Omega}\left|\nabla u_{m}\right|^{2}+u_{m} B u_{m}-\lambda u_{m}^{2} \mathrm{~d} x- \\
-\frac{1}{2^{*}} \int_{\Omega} W(x)\left|u_{m}\right|^{2^{*}} \mathrm{~d} x-\theta \int_{\Omega}\left|\nabla u_{m}\right|^{2}+u_{m} B u_{m}-\lambda u_{m}^{2} \mathrm{~d} x+\theta \int_{\Omega} W(x)\left|u_{m}\right|^{2^{*}} \mathrm{~d} x \geq \\
\geq\left(\frac{1}{2}-\theta\right) \int_{\Omega}\left|\nabla u_{m}\right|^{2}+u_{m} B u_{m}-\lambda u_{m}^{2} \mathrm{~d} x+\left(\theta-\frac{1}{2^{*}}\right) \int_{\Omega} W(x)\left|u_{m}\right|^{2^{*}} \mathrm{~d} x \geq \\
\geq\left(\frac{1}{2}-\theta\right)\left\|u_{m}\right\|^{2}-C_{1} \lambda\left\|u_{m}\right\|_{2^{*}}^{2}+\eta\left(\theta-\frac{1}{2^{*}}\right)\left\|u_{m}\right\|_{2^{*}}^{2^{*}} \geq \\
\geq\left(\frac{1}{2}-\theta\right)\left\|u_{m}\right\|^{2}+\inf _{\rho \geq 0}\left[\eta\left(\theta-\frac{1}{2^{*}}\right) \rho^{2^{*}}-C_{1} \lambda \rho^{2}\right],
\end{gathered}
$$

where $C_{1}>0$ is a positive constant. 
Then $\left(u_{m}\right)_{m \geq 1}$ is bounded in $H_{0}^{1}(\Omega)$. Hence we may extract a subsequence $\left(u_{m}\right)_{m \geq 1}$ (relabeled) such that

$$
\begin{aligned}
& u_{m} \rightarrow u \text { weakly in } H_{0}^{1}(\Omega) \\
& u_{m} \rightarrow u \text { strongly in } L^{p}(\Omega), \text { for any } p \in\left[1,2^{*}\right) \\
& u_{m} \rightarrow u \text { a.e. in } \Omega
\end{aligned}
$$

Now, we prove that $u$ is a solution of $\left(P^{\prime}\right)$. Let $\varphi \in C_{0}^{\infty}(\Omega)$. Then $\left|\mathrm{d} I_{\lambda}(u) \varphi\right| \leq\left\|\mathrm{d} I_{\lambda}\left(u_{m}\right)\right\|_{H^{-1}}\|\varphi\|+\left|\left(\mathrm{d} I_{\lambda}(u)-\mathrm{d} I_{\lambda}\left(u_{m}\right)\right) \varphi\right| \rightarrow 0$, as $m \rightarrow \infty$.

Hence $u$ weakly solves $\left(P^{\prime}\right)$.

Let $v_{m}=u_{m}-u$. Clearly

$$
\begin{aligned}
& v_{m} \rightarrow 0 \text { weakly in } H_{0}^{1}(\Omega) \\
& v_{m} \rightarrow 0 \text { strongly in } L^{p}(\Omega), \text { for any } p \in\left[1,2^{*}\right) \\
& v_{m} \rightarrow 0 \text { a.e. in } \Omega
\end{aligned}
$$

From (2) and (3) observe that

$$
\begin{aligned}
o(1) & =\mathrm{d} I_{\lambda}\left(u_{m}\right) v_{m}=\int_{\Omega} \nabla u_{m} \nabla v_{m}+v_{m} B u_{m}-\lambda u_{m} v_{m} \mathrm{~d} x- \\
& -\int_{\Omega} W(x) v_{m} u_{m}\left|u_{m}\right|^{2^{*}-2} \mathrm{~d} x \\
& =\int_{\Omega}\left|\nabla v_{m}\right|^{2}+v_{m} B v_{m} \mathrm{~d} x-\int_{\Omega} W(x) v_{m} u_{m}\left|u_{m}\right|^{2^{*}-2} \mathrm{~d} x+o(1) \\
& =\left\|v_{m}\right\|_{B}^{2}-\int_{\Omega} W(x) v_{m} u_{m}\left|u_{m}\right|^{2^{*}-2} \mathrm{~d} x+o(1) .
\end{aligned}
$$

Hence

$$
\left\|v_{m}\right\|_{B}^{2}=\int_{\Omega} W(x) v_{m} u_{m}\left|u_{m}\right|^{2^{*}-2} \mathrm{~d} x+o(1) \leq\|W\|_{\infty} \int_{\Omega}\left|v_{m}\right|^{2^{*}} \mathrm{~d} x+o(1)
$$

Since

$$
\mathrm{d} I_{\lambda}\left(u_{m}\right) u_{m}=o(1)
$$


we have that

$$
\int_{\Omega} W(x)\left|u_{m}\right|^{2^{*}} \mathrm{~d} x=\int_{\Omega}\left|\nabla u_{m}\right|^{2}+u_{m} B u_{m}-\lambda u_{m}^{2} \mathrm{~d} x+o(1) .
$$

Using this last equality we obtain

$$
\begin{aligned}
I_{\lambda}\left(u_{m}\right) & =\frac{1}{2}\left(\left\|u_{m}\right\|_{B}^{2}-\lambda\left\|u_{m}\right\|_{2}^{2}\right)-\frac{1}{2^{*}} \int_{\Omega} W(x)\left|u_{m}\right|^{2^{*}} \mathrm{~d} x \geq \\
& \geq \frac{\eta}{N}\|u\|_{2^{*}}^{2^{*}}+\frac{1}{N}\left\|v_{m}\right\|_{B}^{2}+o(1) \geq \frac{1}{N}\left\|v_{m}\right\|_{B}^{2}+o(1) .
\end{aligned}
$$

Then

$\left\|v_{m}\right\|_{B}^{2} \leq N I_{\lambda}\left(u_{m}\right)+o(1)<S_{B}^{N / 2}\|W\|_{\infty}^{\frac{N-2}{2}}$, for $m$ sufficiently large.

From (4) we have

$$
\begin{aligned}
& \left\|v_{m}\right\|_{B}^{2} \leq\|W\|_{\infty} S_{B}^{-\frac{2^{*}}{2}}\left\|v_{m}\right\|_{B}^{2^{*}}+o(1) \Longleftrightarrow \\
& \left\|v_{m}\right\|_{B}^{2}\left(S_{B}^{\frac{2^{*}}{2}}-\|W\|_{\infty}\left\|v_{m}\right\|_{B}^{2^{*}-2}\right) \leq o(1) .
\end{aligned}
$$

Since, from (5),

$$
S_{B}^{\frac{2^{*}}{2}}>\|W\|_{\infty}\left\|v_{m}\right\|_{B}^{2^{*}-2} \text { for } m \text { large enough, }
$$

we obtain that

$$
v_{m} \rightarrow 0, \text { strongly in } H_{0}^{1}(\Omega), \text { as } m \rightarrow \infty,
$$

and this ends the proof of the fact that $I_{\lambda}$ satisfies the Palais-Smale condition on $\left(-\infty, 1 N S_{B}^{N / 2}\|W\|_{\infty}^{\frac{N-2}{2}}\right)$.

\section{Step 2.}

Set

$$
H_{1}=\overline{\widehat{\lambda_{j}} \geq \widehat{\lambda_{+}} \oplus M\left(\widehat{\lambda_{j}}\right)} \text { and } H_{2}=\widehat{\lambda_{j}} \leq \widehat{\lambda_{+}} \oplus M\left(\widehat{\lambda_{j}}\right)
$$


where $M\left(\widehat{\lambda_{j}}\right)$ denotes the eigenspace of $T$ corresponding to the eigenvalue $\widehat{\lambda_{j}}$. Denote $\beta_{\lambda}=H_{2} \sup I_{\lambda}$ and observe that, if $u=\sum_{\widehat{\lambda_{i}} \leq \widehat{\lambda_{+}}} a_{i} \varphi_{i} \in$ $\mathrm{H}_{2}$, we have

$$
\begin{aligned}
I_{\lambda}(u)= & \frac{1}{2}\|u\|_{B}^{2}-\lambda\|u\|_{2}^{2}-\frac{1}{2^{*}} \int_{\Omega} W(x)|u|^{2^{*}} \mathrm{~d} x \leq \frac{1}{2}\left(\widehat{\lambda_{+}}-\lambda\right) \\
& \int_{\Omega} u^{2} \mathrm{~d} x-\frac{\eta}{2^{*}}\|u\|_{2^{*}}^{2^{*}} \leq \frac{1}{2}\left(\widehat{\lambda_{+}}-\lambda\right)(\text { meas }(\Omega))^{2 / N}\|u\|_{2^{\circ}}^{2}-\frac{\eta}{2^{*}}\|u\|_{2^{*}}^{2^{*}} \\
\leq & \rho \geq 0 \sup \left[\frac{1}{2}\left(\widehat{\lambda_{+}}-\lambda\right)(\operatorname{meas}(\Omega))^{2 / N} \rho^{2}-\frac{\eta}{2^{*}} \rho^{2^{*}}\right] \\
= & \frac{1}{N} \eta^{\frac{3-N}{2}}\left(\widehat{\lambda_{+}}-\lambda\right)^{N / 2}(\text { meas }(\Omega)) .
\end{aligned}
$$

Thus

$$
\beta_{\lambda} \leq \frac{1}{N} \eta^{\frac{2-N}{2}}\left(\widehat{\lambda_{+}}-\lambda\right)^{N / 2}(\text { meas }(\Omega)) .
$$

If $u=\sum_{\widehat{\lambda_{i}} \geq \widehat{\lambda_{+}}} a_{i} \varphi_{i} \in H_{1}$, a simple computation shows that

$$
I_{\lambda}(u) \geq\left(1-\frac{\lambda}{\widehat{\lambda_{+}}}\right)\|u\|_{B}^{2}-C_{2}\|u\|_{B}^{2^{*}},
$$

where $C_{2}>0$ is a positive constant. Clearly, there exist constants $\rho_{\lambda}, \xi_{\lambda} \in\left(0, \beta_{\lambda}\right)$ such that

$$
I_{\lambda}(u) \geq \xi_{\lambda}, \text { for any } u \in H_{1},\|u\|_{B}=\rho_{\lambda} .
$$

\section{Step 3.}

Now, it is easy to observe that the hypothesis of Theorem 2 are satisfied for $H=H_{0}^{1}(\Omega), f=I_{\lambda}, \beta=1 N S_{B}^{N / 2}\|W\|_{\infty}^{\frac{N-2}{2}}, V=H_{1}$, $W=H_{2}, \xi=\xi_{\lambda}, \rho=\rho_{\lambda}, \beta^{\prime}=\beta_{\lambda}$ and so, for

$$
\widehat{\lambda_{+}}-\lambda<\left(\frac{\eta}{\|W\|_{\infty}}\right)^{\frac{2}{2^{*}}} S_{B}[\operatorname{meas}(\Omega)]^{-2 / N}
$$


the problem $\left(P^{\prime}\right)$ admits at least

$$
m=\operatorname{dim}\left(H_{1} \cap H_{2}\right)-\operatorname{codim}\left(H_{1}+H_{2}\right)=\operatorname{dim} M\left(\widehat{\lambda_{+}}\right)
$$

pairs of nontrivial solutions

$$
\left\{u_{k}(\lambda),-u_{k}(\lambda)\right\}, k=1,2, \ldots, m
$$

Since

$I_{\lambda}\left(u_{k}(\lambda)\right) \in\left[\delta, \beta^{\prime}\right]$ and $\beta^{\prime} \leq \frac{1}{N} \eta^{\frac{2-N}{2}}\left(\widehat{\lambda_{+}}-\lambda\right)^{N / 2}($ meas $(\Omega)) \rightarrow 0$, as $\lambda \nearrow \widehat{\lambda_{+}}$,

we obtain that

$$
I_{\lambda}\left(u_{k}(\lambda)\right) \rightarrow 0, \text { as } \lambda \nearrow \widehat{\lambda_{+}}, \forall k \in\{1,2, \ldots, m\} .
$$

From this and from $\mathrm{d} I_{\lambda}\left(u_{k}(\lambda)\right)=0$, we obtain that

$$
u_{k}(\lambda) \rightarrow 0, \text { strongly in } H_{0}^{1}(\Omega), \text { as } \lambda \nearrow \widehat{\lambda_{+}} .
$$

since $I_{\lambda}$ satisfies the (PS) condition in the interval

$$
\left(-\infty, 1 N S_{B}^{N / 2}\|W\|_{\infty}^{\frac{N-2}{2}}\right)
$$

Now, from the equivalence between $\left(P^{\prime}\right)$ and $(P)$, it is easy to observe that if $\widehat{\lambda_{+}}-\lambda<\left(\eta\|W\|_{\infty}\right)^{\frac{2}{2^{*}}} S_{B}[\text { meas }(\Omega)]^{-2 / N}$, then (P) admits at least $m$ pairs of nontrivial solutions $\left\{\left(u_{k}(\lambda), v_{k}(\lambda)\right) ;\left(-u_{k}(\lambda),-v_{k}(\lambda)\right)\right\}$, $k=1,2, \ldots, m$, where $v_{k}(\lambda)=1 k B\left(u_{k}(\lambda)\right)$. Moreover, from (6) and the continuity of $B$, we also obtain that

$$
v_{k}(\lambda) \rightarrow 0, \text { strongly in } H_{0}^{1}(\Omega), \text { as } \lambda \nearrow \widehat{\lambda_{+}} .
$$

and this ends the proof.

Acknowledgments. This work done partly while the author was visiting the Department of Applied Mathematics of the Universidad Complutense de Madrid. The author would like to express his gratitude to E. Zuazua for his kind invitation. He is also indebted to the Department for its hospitality and support. 


\section{References}

[BBF] P.Bartolo, V.Benci and D.Fortunato, Abstract critical point theorems and applications to some nonlinear problems with "strong resonance" at infinity, Nonlinear Anal.T.M.A. 7(1983), 981-1012.

[CF] R.Chiappinelli and D.G. de Figueiredo, Bifurcation from infinity and multiple solutions for an elliptic system, Relatorio de Pesquisa (junho-1992), Univ.Estadual de Campinas, Brasil.

[CFP] A.Capozzi, D.Fortunato and G.Palmieri, An existence result for nonlinear elliptic problems involving critical Sobolev exponent, Ann.Inst.Henri Poincare 2(1985), 463-470.

[CFS] G.Cerami, D.Fortunato and M.Struwe, Bifurcation and multiplicity results for nonlinear elliptic problems involving critical Sobolev exponents, Ann.Inst.Henri Poincare 1(1984), 341-350.

[FM] D.G. de Figueiredo and E.Mitidieri, A maximum principle for an elliptic system and applications to semilinear problems, SIAM J.Math.Anal. 17(1986), 836-849.

[LM] A.C.Lazer and P.J.McKenna, On steady state solutions of a system of reaction-diffusion equations from biology, Nonlinear Anal.T.M.A. 6(1982), 523-530.

[P] S.J.Pohozaev, Eigenfunctions of the equation $\Delta u+\lambda f(u)=0$, Soviet Math. Doklady 6(1965), 1408-1411.

[R] F.Rothe, Global existence of branches of stationary solutions for a system of reaction diffusion equations from biology, Nonlinear Anal.T.M.A. 5(1981), 487-498.

[RM] F.Rothe and P. P.Mottoni, A Simple System of ReactionDiffusion Equation Describing Morphogenesis: Asymptotic Behavior, Annali Mat.Pura Appl. 122(1979), 141-157.

[NT] C.P.Niculescu and N.Tarfulea, Solvability of an elliptic system with discontinuous nonlinearity and $L^{1}$ data, Comm.Appl.Nonlinear Analysis (in press). 
[S] E.A. de B. e Silva, Existence and multiplicity of solutions for semilinear elliptic systems, NoDEA 1(1994), 339-363.

Nicolae Tarfulea

Recibido: 12 de Febrero de 1997

Department of Mathematics, Revisado: 7 de Mayo de 1998

University of Craiova,

1100 Craiova,

Romania 